\title{
Image Processing Using the Quaternion Wavelet Transform
}

\author{
Eduardo Bayro-Corrochano and Miguel Angel de La Torre Gomora \\ Computer Science Department, GEOVIS Laboratory \\ Centro de Investigación y de Estudios Avanzados \\ CINVESTAV, Guadalajara, Jalisco 44550, Mexico \\ edb@gdl. cinvestav.mx
}

\begin{abstract}
The contribution of this work is to generalize the real and complex wavelet transforms and to derive for the first time a quaternionic wavelet pyramid for multi-resolution analysis using three phases. The paper can be very useful for researchers and practitioners interested in understanding and applications of the quaternion wavelet transform.
\end{abstract}

\section{Introduction}

This work presents the basics and the practicalities of the Quaternion Wavelet Transform (QWT). Along the lines of Mallat [7,6], we developed a pyramidal model to disentangle symmetries of $2 \mathrm{D}$ signals (images) in the quaternion algebra, getting for each level one approximation and three decompositions (horizontal, vertical and diagonal). Different to the complex wavelet transform, the quaternionic wavelet transform provides three phases for a powerful image analysis while using the phase concept.

In the literature $[8,10]$ the QWT belongs to the theoretical arena where the quaternion mother wavelet is the Haar function, a very inefficient kernel. In our approach we use the more efficient quaternion Gabor kernel and we introduce the multiresolution analysis using the quaternion phase concept. In this regard modulated quaternion Gabor filters are used for computing a pyramid of phases with different resolutions. In the experimental part we show a hierarchical regionbased matching algorithm using discrete QWT to estimate optical flow.

\section{Quaternions}

The quaternion algebra $\mathbb{H}$ was invented by W. R. Hamilton in 1843 [4, 1]. It is an associative non-commutative four-dimensional algebra

$$
\mathbb{H}=\{\boldsymbol{q}=s+x \boldsymbol{i}+y \boldsymbol{j}+z \boldsymbol{k} \mid s, x, y, z \in \mathbb{R}\},
$$

where the orthogonal imaginary numbers $\boldsymbol{i}, \boldsymbol{j}$ and $\boldsymbol{k}$ obey the following multiplicative rules

$$
\boldsymbol{i}^{2}=\boldsymbol{j}^{2}=-1, \boldsymbol{k}=\boldsymbol{i} \boldsymbol{j}=-\boldsymbol{j} \boldsymbol{i} \rightarrow \boldsymbol{k}^{2}=-1
$$


The conjugated of a quaternion is given by

$$
\overline{\boldsymbol{q}}=s-x \boldsymbol{i}-y \boldsymbol{j}-z \boldsymbol{k}
$$

For the quaternion $\boldsymbol{q}$ we can compute its partial angles as $\arg _{i}(q)=s \tan 2(x$, $s), \arg _{j}(q)=s \tan 2(y, s), \arg _{k}(q)=s \tan 2(z, s)$, and its partial modules and its projections on its imaginary axes as $\bmod _{i}(q)=\sqrt{s^{2}+x^{2}}, \bmod _{j}(s)=\sqrt{s^{2}+y^{2}}$, $\bmod _{k}(q)=\sqrt{s^{2}+z^{2}}, \bmod _{i}(q) \exp \left(i \arg _{i}(q)\right)=s+x i, \bmod _{i}(q) \exp \left(j \arg _{j}(q)\right)=$ $s+y j$ and $\bmod _{k}(q) \exp \left(k \arg _{k}(q)\right)=s+z k$.

In a similar way as the complex numbers which can be expressed in a polar representation, we can also represent a quaternion $\boldsymbol{q}=r+x \boldsymbol{i}+y \boldsymbol{j}+z \boldsymbol{k}$ in a polar form

$$
\boldsymbol{q}=|\boldsymbol{q}| e^{\boldsymbol{i}_{\phi}} e^{\boldsymbol{k} \psi} e^{\boldsymbol{j} \theta}
$$

where the phases ranges are delimited as follows $(\phi, \theta, \psi) \in\left[-\pi, \pi\left[\times\left[-\frac{\pi}{2}, \frac{\pi}{2}[\times\right.\right.\right.$, $\pi\left[-\times\left[-\frac{\pi}{4}, \frac{\pi}{4}\right]\right.$.

For a unit quaternion $\boldsymbol{q}=q_{0}+q_{x} i+q_{y} j+q_{z} k,|\boldsymbol{q}|=\boldsymbol{q} \overline{\boldsymbol{q}}=1$, its phase can be evaluated first by computing

$$
\psi=-\frac{\arcsin \left(2\left(q_{x} q_{y}-q_{0} q_{z}\right)\right)}{2}
$$

and then by checking that it adheres to the following rules:

- If $\psi \in]-\frac{\pi}{4}, \frac{\pi}{4}\left[\right.$, then $\phi=\frac{\arg \left(\boldsymbol{q} \mathcal{T}_{j}(\overline{\boldsymbol{q}})\right)}{2}$ and $\theta=\frac{\arg \left(\mathcal{T}_{i}(\overline{\boldsymbol{q}}) \boldsymbol{q}\right)}{2}$.

- If $\psi= \pm \frac{\pi}{4}$, then select either $\phi=0$ and $\theta=\frac{\arg \left(\mathcal{T}_{k}(\overline{\boldsymbol{q}}) \boldsymbol{q}\right)}{2}$ or $\theta=0$ and $\phi=$ $\frac{\arg \left(\boldsymbol{q} \mathcal{T}_{k}(\overline{\boldsymbol{q}})\right)}{2}$.

- If $e^{\boldsymbol{i} \phi} e^{\boldsymbol{k}_{\psi}} e^{\boldsymbol{j} \theta}=-\boldsymbol{q}$ and $\phi \geq 0$, then $\phi \rightarrow \phi-\pi$.

- If $e^{\boldsymbol{i}_{\phi}} e^{\boldsymbol{k} \psi} e^{\boldsymbol{j} \theta}=-\boldsymbol{q}$ and $\phi<0$, then $\phi \rightarrow \phi+\pi$,

where we use the three nontrivial involutions $[3] \mathcal{T}_{\boldsymbol{i}}(\boldsymbol{q})=-\boldsymbol{i} \boldsymbol{q} \boldsymbol{i}=q_{0}+q_{x} \boldsymbol{i}-q_{y} \boldsymbol{j}-$ $q_{z} \boldsymbol{k}, \mathcal{T}_{\boldsymbol{j}}(\boldsymbol{q})=-\boldsymbol{j} \boldsymbol{q} \boldsymbol{j}=q_{0}-q_{x} \boldsymbol{i}+q_{y} \boldsymbol{j}-q_{z} \boldsymbol{k}$, and $\mathcal{T}_{\boldsymbol{k}}(\boldsymbol{q})=-\boldsymbol{k} \boldsymbol{q} \boldsymbol{k}=q_{0}-q_{x} \boldsymbol{i}-$ $q_{y} \boldsymbol{j}+q_{z} \boldsymbol{k}$.

\section{Quaternion Wavelet Transform}

The quaternion wavelet transform is a natural extension of the real and complex wavelet transform taking into account the axioms of the quaternion algebra, the quaternionic analytic signal [2] and the separability propriety. The QWT is applied for signals of 2-D or bigger dimensions.

The multi-resolution analysis can be also straightforwardly extended to the quaternionic case; we can improve therefore the power of the phase concept which in the real wavelets is not possible and in the case of the complex is limited to only one phase. Thus, in contrast to the similarity distance used in the complex wavelet pyramid [9], we favor the quaternionic phase concept for top-down parameter estimation. 
For the quaternionic versions of the wavelet scale function $h$ and the wavelet function $g$ we choose two quaternionic modulated Gabor filters in quadrature as follows

$$
\begin{aligned}
& h^{q}=g\left(x, y, \sigma_{1}, \varepsilon\right) \exp \left(\mathbf{i} \frac{c_{1} \omega_{1} x}{\sigma_{1}}\right) \exp \left(\mathbf{j} \frac{c_{2} \varepsilon \omega_{2} y}{\sigma_{1}}\right)=h_{e e}^{q}+h_{o e}^{q} \mathbf{i}+h_{e o}^{q} \mathbf{j}+h_{o o}^{q} \mathbf{k}, \\
& g^{q}=g\left(x, y, \sigma_{2}, \varepsilon\right) \exp \left(\mathbf{i} \frac{\tilde{c}_{1} \tilde{\omega}_{1} x}{\sigma_{2}}\right) \exp \left(\mathbf{j} \frac{\tilde{c}_{2} \varepsilon \tilde{\omega}_{2} y}{\sigma_{2}}\right)=g_{e e}^{q}+g_{o e}^{q} \mathbf{i}+g_{e o}^{q} \mathbf{j}+g_{o o}^{q} \mathbf{k} .
\end{aligned}
$$

Note that the horizontal axis $x$ is related with $\mathbf{i}$ and the vertical axis $y$ is related with $\mathbf{j}$, both imaginary numbers of the quaternion algebra fulfill the equation $\mathbf{k}=\mathbf{j i}$.

The right parts of the equations (6) and (7) obey a natural decomposition of a quaternionic analytic function: the subindex oo stands for even-even a symmetric filter, eo even-odd or oe odd-even both stand for unsymmetrical filters and oo odd-odd stand for a unsymmetrical filter as well. Thus we can see clearly that $h^{q}$ and $g^{q}$ of equations (6) and (7) are powerful filters for disentanglement of the symmetries of the 2-D signals. It is clear that for exploting the phase concept, complex wavelets can use only one phase, where as the quaternionic wavelets offer three phases.

\subsection{Quaternionic Wavelet Pyramid}

For the 2-D image function $f(x, y)$, a quaternionic wavelet multi-resolution will be written as

$$
f(x, y)=A_{n}^{q} f+\sum_{j=1}^{n}\left[D_{j, 1}^{q} f+D_{j, 2}^{q} f+D_{j, 3}^{q} f\right] .
$$

The upper index $q$ stands for indicating quaternion 2-D signal. We can characterize each approximation function $A_{j}^{q} f(x, y)$ and the difference components $D_{j, p}^{q} f(x, y)$ for $p=1,2,3$ by means of a $2 \mathrm{D}$ scaling function $\boldsymbol{\Phi}^{q}(x, y)$ and its associate wavelet functions $\boldsymbol{\Psi}_{p}^{q}(x, y)$ as follows

$$
\begin{aligned}
A_{j}^{q} f(x, y) & =\sum_{k=-\infty}^{+\infty} \sum_{l=-\infty}^{+\infty} a_{j, k, l} \boldsymbol{\Phi}_{j, k, l}^{q}(x, y), \\
D_{j, p}^{q} f(x, y) & =\sum_{k=-\infty}^{+\infty} \sum_{l=-\infty}^{+\infty} d_{j, p, k, l} \boldsymbol{\Psi}_{j, p, k, l}^{q}(x, y)
\end{aligned}
$$

where

$$
\begin{aligned}
\boldsymbol{\Phi}_{j, k, l}^{q}(x, y) & =\frac{1}{2^{j}} \boldsymbol{\Phi}^{q}\left(\frac{x-k}{2^{j}}, \frac{y-l}{2^{j}}\right), \quad(j, k, l) \in \mathbb{Z}^{3}, \\
\boldsymbol{\Psi}_{j, p, k, l}^{q}(x, y) & =\frac{1}{2^{j}} \boldsymbol{\Psi}_{p}^{q}\left(\frac{x-k}{2^{j}}, \frac{y-l}{2^{j}}\right)
\end{aligned}
$$


and

$$
a_{j, k, l}(x, y)=<f(x, y), \boldsymbol{\Phi}_{j, k, l}^{q}(x, y)>, d_{j, p, k, l}=<f(x, y), \boldsymbol{\Psi}_{j, p, k, l}^{q}(x, y)>.
$$

In order to carry out a separable quaternionic multi-resolution analysis, we decompose the scaling function $\boldsymbol{\Phi}^{q}(x, y)_{j}$ and the wavelet functions $\boldsymbol{\Psi}_{p}^{q}(x, y)_{j}$ for each level $j$ as follows

$$
\begin{aligned}
& \boldsymbol{\Phi}^{q}(x, y)_{j}=\phi^{\boldsymbol{i}}(x)_{j} \phi^{\boldsymbol{j}}(y)_{j}, \\
& \boldsymbol{\Psi}_{1}^{q}(x, y)_{j}=\phi^{\boldsymbol{i}}(x)_{j} \psi^{\boldsymbol{j}_{(y)}}, \mathbf{\Psi}_{2}^{q}(x, y)_{j}=\psi^{\boldsymbol{i}}(x)_{j} \phi^{\boldsymbol{j}}(y)_{j}, \\
& \boldsymbol{\Psi}_{3}^{q}(x, y)_{j}=\psi^{\boldsymbol{i}}(x)_{j} \psi^{\boldsymbol{j}}(y)_{j},
\end{aligned}
$$

where $\phi^{\boldsymbol{i}}(x)_{j}, \psi(x)_{j}^{\boldsymbol{i}}$ are 1-D complex filters applied along the rows and columns respectively. Note that in the $\phi$ and $\psi$ we use the imaginary number $\boldsymbol{i}, \boldsymbol{j}$ of quaternions that fulfill $\boldsymbol{j i}=\boldsymbol{k}$. By using these formulas we can build quaternionic wavelet pyramid. Figure 1 shows the two primary levels of the pyramid (fine to coarse). According the equation (16) the approximation after the first level $A_{1}^{q} f(x, y)$ is the output of $\boldsymbol{\Phi}^{q}(x, y)_{1}$, the differences $D_{1,1}^{q} f, D_{1,2}^{q} f, D_{1,3}^{q} f$ are the outputs of $\boldsymbol{\Psi}_{1,1}^{q}(x, y), \boldsymbol{\Psi}_{1,2}^{q}(x, y)$ and $\boldsymbol{\Psi}_{1,3}^{q}(x, y)$. The procedure continues through the $j$ levels decimating the image at the outputs of the levels (indicated in the Figure 1 within a circle).

The quaternionic wavelet analysis from level $j-1$ to level $j$ corresponds to the transforming of one quaternionic approximation to a new quaternionic approximation and three quaternionic differences, i.e.

$$
\left\{A_{j-1}^{q}\right\} \rightarrow\left\{A_{j}^{q}, D_{j, p}^{q}, p=1,2,3\right\},
$$

compare that we do not use the idea of mirror tree [5]. As a result, the quaternionic wavelet tree is the compact and economic processing structure to be used for the case of n-D multi-resolution analysis.

The procedure of quaternionic wavelet multi-resolution analysis depicted partially in Figure 1 is as follows

- convolve the 2-D real signal at level $j$ and convolve it with the scale and wavelet filters $H_{j}^{q}$ and $G_{j}^{q}$ along the rows of the the 2-D signal.

- $H_{j}^{q}$ and $G_{j}^{q}$ are convolved with the columns of the previous responses of the filters $H_{j}^{q}$ and $G_{j}^{q}$.

- Subsample the responses of these filters by a factor of two $(\downarrow 2)$.

- The real part of the approximation at level $\mathrm{j}$ is taken as input at the next level $j$, this process continues through the all levels $j=1, \ldots, n$ repeating the steps $1 \rightarrow 4$.

\section{Applications}

The estimation of the disparity using the concept of local phase begins with the assumption that a couple of successive images are related as follows

$$
f_{1}(x)=f_{2}(x+\mathbf{d}(x)),
$$




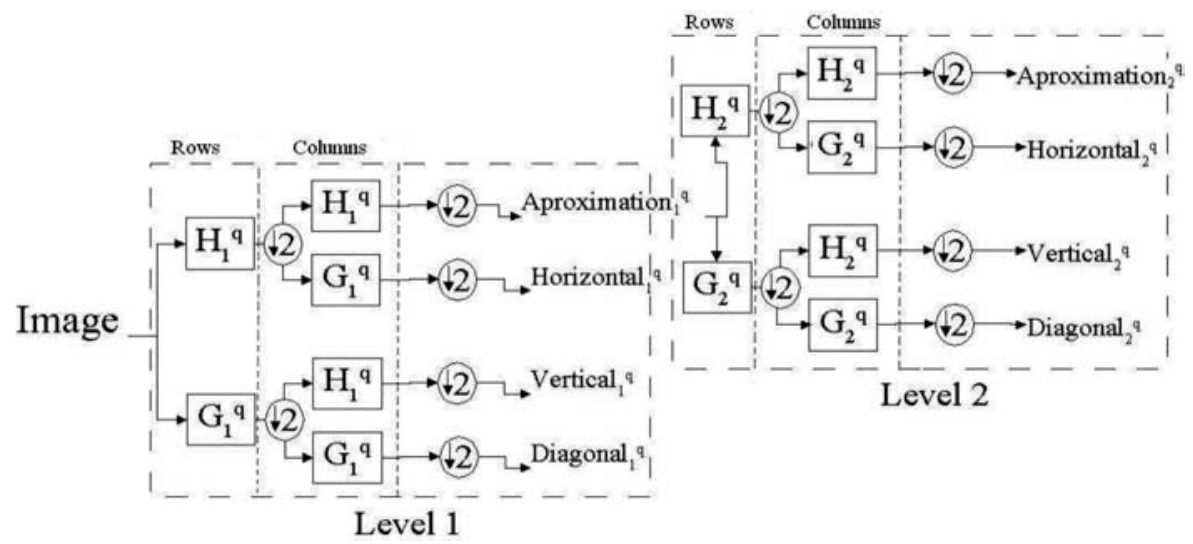

Fig. 1. Abstraction of two levels of the quaternionic wavelet pyramid

where $\mathbf{d}(x)$ is the unknown vector. Assuming that the phase varies linearly (here we see the importance of shifting invariant filters), the displacement $\mathbf{d}(x)$ can be computed as

$$
d_{x}(x)=\frac{\phi_{2}(x)-\phi_{1}(x)+n(2 \pi+k)}{2 \pi u_{r e f}}, \quad d_{y}(x)=\frac{\theta_{2}(x)-\theta_{1}(x)+m \pi}{2 \pi v_{r e f}},
$$

with reference frequencies $\left(u_{r e f}, v_{r e f}\right)$ that are not known a priori. Here $\phi(x)$ and $\theta(x)$ are the first two components of the quaternionic local phase of the quaternionic filter. We choose $n, m \in \mathbb{Z}$, so that $d_{x}$ and $d_{y}$ are within a valid range. Depending on $m, k$ is defined as

$$
k= \begin{cases}0, \text { if } m \text { is even } \\ 1, \text { if } m \text { is odd }\end{cases}
$$

A good disparity estimation is achieved if $\left(u_{r e f}, v_{r e f}\right)$ are well chosen. There are two methods of dealing with the problem: i) the constant model where $u_{r e f}$ and $v_{r e f}$ are chosen as the central frequencies of the filters; ii) the model for the complex case called the local model, it supposes that the phase takes the same value $\Phi_{1}(x)=\Phi_{2}(x+d)$ in two correspondent points in both images, thus one estimates $d$ by approximating $\Phi_{2}$ via a first order Taylor's series expansion about $\mathbf{x}$ :

$$
\Phi_{2}(\mathbf{x}+\mathbf{d}) \approx \phi_{2}(\mathbf{x})+(\mathbf{d} \cdot \nabla) \phi_{2}(\mathbf{x})
$$

where we call $\Phi=(\phi, \theta)$. Solving equation (21) for $\mathbf{d}$ we obtain the estimated disparity of the local model. In our experiments we assume that $\phi$ varies along the $\mathbf{x}$ direction and $\theta$ along $y$. Using this assumption the disparity (equation (19)) can be estimated using the following reference frequencies

$$
u_{\text {ref }}=\frac{1}{2 \pi} \frac{\partial \phi_{1}}{\partial x}(x), \quad v_{\text {ref }}=\frac{1}{2 \pi} \frac{\partial \theta_{1}}{\partial y}(x) .
$$


If locations where $u_{\text {ref }}$ and $v_{r e f}$ are equal to zero, the equations (22) are undefined. One can neglect these localities using a sort of confidence mask.

As an application we will show the estimation of the optical flow of the Rubik cube image sequences. We used the following scaling and wavelet quaternionic filters

$$
\begin{aligned}
& h^{q}=g\left(x, \sigma_{1}, \varepsilon\right) \exp \left(i \frac{c_{1} \omega_{1} x}{\sigma_{1}}\right) \exp \left(j \frac{c_{2} \varepsilon \omega_{2} y}{\sigma_{1}}\right) \\
& g^{q}=g\left(x, \sigma_{2}, \varepsilon\right) \exp \left(i \frac{\tilde{c}_{1} \tilde{\omega}_{1} x}{\sigma_{2}}\right) \exp \left(j \frac{\tilde{c}_{2} \varepsilon \tilde{\omega}_{2} y}{\sigma_{2}}\right)
\end{aligned}
$$

with $\sigma_{1}=\frac{\pi}{6}$ y $\sigma_{2}=\frac{5 \pi}{6}$ so that the filters are in quadrature and $c_{1}=\tilde{c}_{1}=3$, $\omega_{1}=1$ y $\omega_{2}=1, \varepsilon=1$. The resulting quaternionic mask will also be sub-sampled through the levels of the pyramid. For the estimation of the the optical flow we use two successive images of the image sequence, thus two quaternionic wavelet pyramids are generated. For our examples we computed 4 levels. According to equation (17) at each level of each pyramid we obtain 16 images accounting for the four quaternionic outputs (approximation $\Phi$ and the details $\Psi_{1}$ (horizontal), $\Psi_{2}$ (vertical), $\Psi_{3}$ (diagonal)). The phases are evaluated according to the equations (2). Figure 2 shows the magnitudes and phases obtained at a level $j$ using two successive Rubik's images.

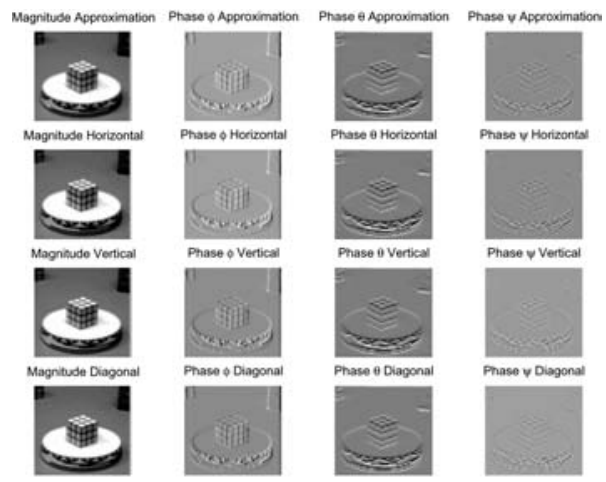

Fig. 2. The magnitudes and phase images for the Rubik's sequence at certain level $j$ : (upper row) the approximation $\Phi$ and (next rows) the details $\Psi_{1}$ (horizontal), $\Psi_{2}$ (vertical), $\Psi_{3}$ (diagonal)

After we have computed the phases, we proceed to estimate the disparity images using the equations (19), where the reference frequencies $u$ and $v$ are calculated according to equation (22). We apply the confidence mask as shown in Figure 3.a.

After the estimation of the disparity has been filtered by the confidence mask, we proceed to estimate the optical flow at each point computing a velocity vector in terms of the horizontal and vertical details. Now, using the information of the diagonal detail, we adjust the final orientation of the velocity vector. Since the 

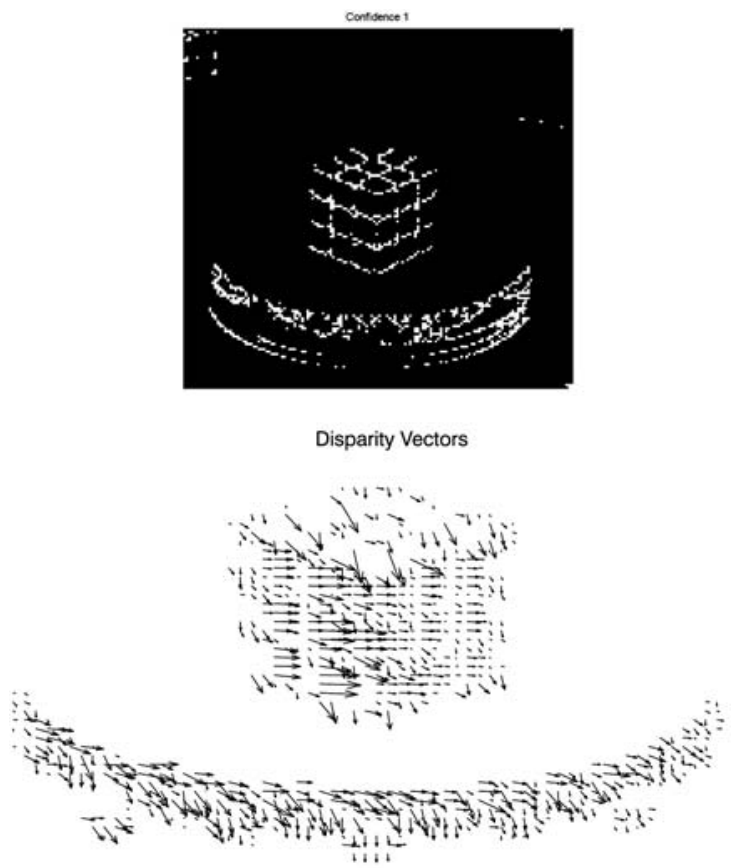

Fig. 3. a) Confidence mask. b) Estimated optical flow

procedure starts from the higher level (top-down) the resulting matrix of the optical flow vectors is expanded in size equal to the next level. The algorithm estimates the optical flow at the new level, the result is compared with the one of the expanded previous level. The velocity vectors of the previous level fill gaps in the new level.

This procedure is continued until the bottom level. In this way the estimation is refined smoothly, the well defined optical flow vectors are passing from level to level increasing the confidence of the vectors at the finest level. It is unavoidable that some artifacts survive at the final stage. A final refinement can be applied imposing a magnitude thresholding and certainly deleting isolated small vectors.

Figure 3 presents the optical flow for an image couple of the Rubik's image sequence. Note that we present all the optical flow vectors that we get with our procedure. We have checked results of some other authors using their own MatLab routines of complex wavelets, and we have detected that their results were presented after deleting many small vectors or the ones in conflicting locations like in the corners of the Rubik's cube.

Our experiment shows that the procedure using quaternionic wavelet pyramid and the phase concept for the parameter estimation work very well.

\section{Conclusion}

This paper presents the theory and practicalities of the quaternion wavelet transform. The Mallat's multi-resolution analysis has been extended using the quater- 
nion wavelets. A big advantage of the approach is that it offers three phases at each level of the pyramid, which can be used for a powerful top-down parameter estimation. The authors believe that the paper can help to push forward the research and applications of hyper-complex wavelet transforms.

\section{Acknowledgments}

The authors were supported by the project 49 of CONACYT-Fondo Sectorial de Investigación en Salud y Seguridad Social.

\section{References}

1. Bayro-Corrochano E., 2001. Geometric Computing for Perception Action Systems, Springer Verlag, Boston.

2. Bülow T., 1999. Hypercomplex Spectral Signal Representations for the Processing and Analysis of Images. PhD. thesis, University Christian Albrechts university of Kiel.

3. Chernov V. M., 1995. Discrete orthogonal transforms with the data representation in composition algebras. In Scandinavian Conference on Image Analysis, Uppsala, Sweden, pp. 357-364.

4. Hamilton W.R., 1969. Elements of Quaternions. Longmans Green, London 1866. Chelsea, New York.

5. Magarey, J.F.A. and Kingsbury, N.G. 1998. Motion estimation using a complexvalued wavelet transform. IEEE Trans. Image Proc. 6, 549-565.

6. S. Mallat A Wavelet Tour of Signal Processing. Academic Press, San Diego, CA, Second Edition, 1998.

7. Mallat S.,1989. A Teory for multiresolution signal decomposition: the wavelet representation. IEEE Trans. Patt. Anal. and Mach. Intell., 11(7):674-693, July.

8. Mitrea M., 1994. Clifford Waveletes, singular Integrals and Hardy spaces. Lecture notes in mathematics 1575, Spinger Verlag.

9. Pan H.-P., 1996. Uniform full information image matching complex conjugate wavelet pyramids. XVIII ISPRS Congress, Viena, July 1996, vol. XXXI.

10. Traversoni L., 2001. Image Analysis using Quaternion Wavelet, in Geometric Algebra in Science and Engineering Book, E. Bayro Corrochano and G. Sobczyk, Springer Velag, Chap. 16. 